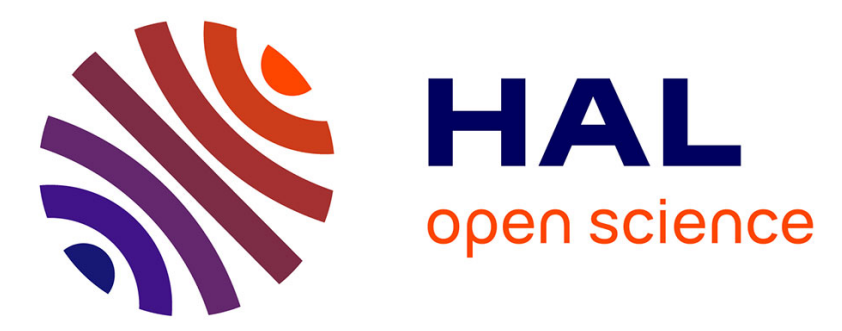

\title{
Delay and state observer for SISO LTI systems
}

Vincent Léchappé, Jesus de Leon, Emmanuel Moulay, Franck Plestan, Alain

\author{
Glumineau
}

\section{To cite this version:}

Vincent Léchappé, Jesus de Leon, Emmanuel Moulay, Franck Plestan, Alain Glumineau. Delay and state observer for SISO LTI systems. American Control Conference, Jul 2015, Chicago, United States. 10.1109/ACC.2015.7172051 . hal-01179410

\section{HAL Id: hal-01179410 https://hal.science/hal-01179410}

Submitted on 26 Jan 2016

HAL is a multi-disciplinary open access archive for the deposit and dissemination of scientific research documents, whether they are published or not. The documents may come from teaching and research institutions in France or abroad, or from public or private research centers.
L'archive ouverte pluridisciplinaire HAL, est destinée au dépôt et à la diffusion de documents scientifiques de niveau recherche, publiés ou non, émanant des établissements d'enseignement et de recherche français ou étrangers, des laboratoires publics ou privés. 


\title{
Delay and state observer for SISO LTI systems
}

\author{
V. Léchappé, J. De León, E. Moulay, F. Plestan and A. Glumineau
}

\begin{abstract}
Index Terms-Input delay, time delay estimation, robust observer.

Abstract-This paper deals with the problem of state and delay estimation for SISO LTI systems, with unknown timevarying delay in the input. Thanks to an adequate approximation of the delayed input by the Taylor's theorem, an original approach based on observer design is proposed in order to estimate both state and delay. This new technique allows the estimation of time-varying delay. The convergence of the observer is formally proved. The efficiency of the method is widely illustrated by simulations.
\end{abstract}

\section{INTRODUCTION}

Input delay systems are a subcategory of time-delay systems (TDS). They especially include all remote controlled devices. The source of delay is multiple: the network configuration (see the extensive literature on networked control system [14], [24]), computational delays or physical transport delays. When the delay is small or the system is open loop stable, delay free controllers can often achieve stabilization. However, predictive techniques are often required as soon as the delay becomes larger and cannot be neglected anymore [18], [23]. To use such methods, the exact value of the delay is needed. However, in real applications, it is quite difficult to measure the delay with precision so it has to be estimated. For an exhaustive review of time delay estimation (TDE) techniques, the reader can refer to the report [20] by O'Dwyer. To build the prediction not only the delay is needed but also all the state. However, standard observation techniques cannot be applied when the delay is unknown. In this paper, both problem are addressed : delay estimation and state observation.

\section{A. Delay identification}

Time delay identification has often been based on a signal processing approach and particularly in the acoustic field [7][16]. These methods are not well adapted in the control context because they are usually offline methods and because they require the knowledge of the delayed signal. A survey of TDE techniques with a signal processing focus is given in [4]. On the contrary, some works use control oriented tools. In these approaches, the delay is considered

This work was supported by the bilateral project Mexico-Francia (PCP 2013) 209488 CONACYT between the Ecole Centrale de Nantes and the Universidad Autónoma de Nuevo León.

Vincent Léchappé, Franck Plestan and Alain Glumineau are with LUNAM Université, Ecole Centrale de Nantes, IRCCyN UMR CNRS 6597

Nantes, France. Firstname. Namedirccyn.ec-nantes.fr.

Emmanuel Moulay is with Xlim, UMR CNRS 7252, Université de

Poitiers emmanuel.moulay@univ-poitiers.fr

Jesús de León is with FIME-UANL, Universidad Autónoma de Nuevo León, Mexico drjleon@gmail.com as a parameter of the system and its identification is often combined to the identification of other parameters. Some authors use the frequency domain where the delay appears as a parameter in the exponential $e^{-h s}$. In [1], the term $e^{-h s}$ is approximated by a rational transfer function of the Padé form; then a standard discrete least-square algorithm is used to minimize an objective function. Tuch et al. [25] also based their approach on the frequency domain and proposed a continuous recursive least square algorithm. However, this method does not work if the initial conditions of the system are not perfectly known. In [19], a PDE approximation is used to extract the delay. In [8], a similar techniques as in [25] is applied but the value of $u(t-h)$ is required. In [9], observers have been used to identify the delay. However, all the state and its time-derivatives are needed; so the method is very sensitive to noise measurement. In [2], a convolution approach is discussed for transfer function systems.

Note that, in all previously mentioned articles the delay is constant.

\section{B. State observation of TDS with unknown delay}

In previous methods, transfer function models are often considered and the problem of state observation is not adressed. On the contrary, some papers deal with the problem of state observation with an unknown delay but do not estimate the delay [11], [21], [22]. The references on that topic are much more scarce ${ }^{1}$. As far as the authors knowledge, the only paper that deals with both delay identification and state reconstruction is [10]. The design of their state observer is largely based on a particular sampling/holding technique.

\section{Contribution}

The main contribution of this paper is to offer an online identification method, based on the theory of robust observation, for both state and delay. The method works for timevarying delays and only requires the knowledge of the input value and the output at time $t$. The observer convergence is formally proven even for time-varying delays.

\section{Paper's structure}

The paper is organized as follows. The problem presentation and an observability study are provided in Section II. Section III is dedicated to the observer construction and the convergence analysis. The results are illustrated by simulations in Section IV and a conclusion and some future developments are given in Section V.

\footnotetext{
${ }^{1}$ However, there are a lot of works on state observation of TDS with known delay (see [11] and references therein).
} 


\section{Problem STATEMENT AND OBSERVABILITy ANALYSIS}

\section{A. Problem statement}

The considered systems are SISO LTI systems with a time varying delay $h(t)$ acting on the control input $u$ :

$$
\left\{\begin{array}{l}
\dot{x}(t)=A x(t)+B u(t-h(t)) \\
y(t)=C x(t) \\
x(0)=x_{0}
\end{array}\right.
$$

with $x \in \mathbb{R}^{n}, u \in \mathbb{R}, A \in \mathbb{R}^{n \times n}, B \in \mathbb{R}^{n \times 1}, C \in \mathbb{R}^{1 \times n}$. This work is focused on the estimation of a single timevarying delay in the input of LTI SISO systems; no parameter identification is considered here.

Assumption 1: The matrices $A, B$ and $C$ are constant and known. The pair $(A, C)$ is observable.

Assumption 2: The relative degree of system (1) is $n$.

Assumption 3: The delay is a unknown and time-varying. It is modeled as a continuous and differentiable function that satisfies $h(t) \in[0, \bar{h}]$. Its dynamics $\dot{h}(t)=\eta(t)$ is unknown and bounded ${ }^{2}$ : for all $t>0,|\eta(t)| \leq H$. The bounds $\bar{h}$ and $H$ can be unknown as well.

Assumption 4: The input $u$ is at least twice time differentiable and the derivatives are bounded for all $t>-\bar{h}$. In particular, there exists $M>0$ such that

$$
|\ddot{u}(t)| \leq M
$$

for all $t \geq-\bar{h}$.

The objective is to design an observer that reconstructs the state $x(t)$ and the delay $h(t)$ from the only knowledge of the output $y(t)$ and the input $u(t)$ and its time-derivatives.

The Taylor's theorem is used to kick the delay out of the control. The input $u$ is differentiable for all $t>-\bar{h}$. Then, there exists a function $\gamma:]-\bar{h} ;+\infty[\rightarrow \mathbb{R}$ such that for all $t^{\prime}>-\bar{h}$

$$
u\left(t^{\prime}\right)=u(t)+\left(t-t^{\prime}\right) \dot{u}(t)+\gamma\left(t^{\prime}\right)
$$

where $\gamma$ is called the remainder. In particular for $t^{\prime}=t-$ $h(t)>-\bar{h}$, it leads to

$$
u(t-h(t))=u(t)-h(t) \dot{u}(t)+\gamma(t-h(t))
$$

for all $t>-\bar{h}$. Besides, since $u$ is twice differentiable from Assumption 4, the remainder $\gamma$ is such that

$$
|\gamma(t-h(t))| \leq \frac{h^{2}(t)}{2} M
$$

From expression (3), the first order approximation of $u(t-$ $h(t))$ is

$$
u(t-h(t)) \approx u(t)-h(t) \dot{u}(t) .
$$

Note that it could be possible to extend the approximation to higher order to make it more accurate. However, from a practical point of view, it has been arbitrarily decided to stop at order one as a tradeoff between problems induced by numerical differentiation and approximation precision.

\footnotetext{
${ }^{2}$ No restriction is made on the delay rate; $H$ can be larger than one (fast-varying delays)
}

By substituting $u(t-h(t))$ by (3) in (1), an extended system with perturbation is obtained:

$$
\left\{\begin{array}{l}
{\left[\begin{array}{l}
\dot{x} \\
\dot{h}
\end{array}\right]=\left[\begin{array}{cc}
A & -B \dot{u} \\
0 & 0
\end{array}\right]\left[\begin{array}{l}
x \\
h
\end{array}\right]+\left[\begin{array}{c}
B \\
0
\end{array}\right] u(t)+\left[\begin{array}{c}
B \gamma(t-h(t)) \\
\eta(t)
\end{array}\right]} \\
y=C x
\end{array}\right.
$$

Denoting the extended vector $X=\left[x^{T} h\right]^{T}, X \in \mathbb{R}^{n+1}$, the system can be rewritten in the general form:

$$
\left\{\begin{array}{l}
\dot{X}=\bar{A}(\dot{u}) X+\bar{B} u(t)+\Gamma(t, t-h(t)) \\
y=Y(X)=\bar{C} X
\end{array}\right.
$$

where

$$
\begin{gathered}
\bar{A}(\dot{u})=\left[\begin{array}{cc}
A & -B \dot{u} \\
0 & 0
\end{array}\right], \bar{B}=\left[\begin{array}{c}
B \\
0
\end{array}\right], \bar{C}=\left[\begin{array}{ll}
C & 0
\end{array}\right] \text { and } \\
\Gamma(t, t-h(t))=\left[\begin{array}{c}
B \gamma(t-h(t)) \\
\eta(t)
\end{array}\right] .
\end{gathered}
$$

It is important to note that the extended system (6) is delayfree, with respect to the input, thanks to the expression (3). However, systems (1) and (6) are equivalent in the sense that they have the same state trajectories. The transformation is only a convenient way to rewrite the system in order to apply existing results from observer literature. The error of approximation $\gamma$ is going to be considered as a perturbation in the design of the observer as well as the dynamics of the delay $\eta$. This is a key point of the method : considering the delay dynamics as perturbation and designing a robust observer that is able to reconstruct the state and the delay in spite of the uncertainty $\Gamma$. In the next part, the observability of the extended system (6) is investigated.

\section{B. Delay observability}

First the following assumption is made:

Assumption 5: The perturbation $\Gamma$ does not modify the observability of (6).

Then, the observability condition for extended system (6) is given in the next theorem. See [13] for observability definitions.

Theorem 1: Extended system (6) is observable if and only if

$$
\dot{u}(t) \neq 0 \quad \forall t>0 .
$$

Proof: First note that from Assumption 2, one has

- $C A^{i} B=0$ for all $i$ such that $0 \leq i \leq n-2$

- $C A^{n-1} B \neq 0$.

Considering $\Gamma=0$ (Assumption (5)), the observation space is defined by

$$
\mathcal{O}(Y)=\operatorname{span}\left\{C x, C A x, \ldots, C A^{n-1} x, C A^{n} x-C A^{n-1} B \dot{u} h\right\} .
$$

Since the initial system is observable, one has

$$
\operatorname{dim} \operatorname{span}\left\{C d x, C A d x, \ldots, C A^{n-1} d x\right\}=n
$$

so

$$
d \mathcal{O}(Y)=\operatorname{span}\left\{C d x, C A d x, \ldots, C A^{n-1} d x,-C A^{n-1} B \dot{u} d h\right\}
$$

and $\operatorname{dim}(d \mathcal{O}(Y))=n+1$ if and only if $C A^{n-1} B \dot{u} \neq 0$. Finally, from [13], system (6) is observable if and only if condition (7) is verified because $C A^{n-1} B \neq 0$. 
This is a logical condition because if the input is constant, the delay as no influence on the system so it cannot be observed. This condition is very restrictive because it means that the input has to be strictly monotonic. However, this condition can be relaxed using the notion of persistence defined in [3]. The definition is recalled below.

Definition 1: A measurable bounded signal $\dot{u}$ is said to be regularly persistent for system (6) if there exists $T>0$, $\alpha>0$ and $t_{0}>0$ such that $\min _{i}\left(\lambda_{i}(W(t, T, \dot{u}))>\alpha\right.$ for all $t>t_{0}$ where $W(t, T, \dot{u})$ is the Observability Gramian and $\lambda_{i}(M)$ denotes the $i^{t h}$ eigenvalue of the matrix $M$.

Roughly speaking, it allows $\dot{u}$ to cancel at some isolated time instants without deteriorating the estimation.

Assumption 6: The signal $\dot{u}$ is regularly persistent.

\section{A NEW SCHEME OF DELAY-STATE OBSERVER}

\section{A. Kalman like observer design [12]}

Kalman-like observer is easy to tune because it only has one parameter to adjust and it is well adapted for linear systems with matrix $A$ depending on an external signal. That is why, it has been chosen in this work. From [12], a Kalmanlike observer for (6) reads as

$$
\dot{\hat{X}}=\bar{A}(\dot{u}) \hat{X}+\bar{B} u-S^{-1} R \bar{C}^{T} \bar{C}(\hat{X}-X)
$$

where the matrix $S$ is the solution of

$$
\left\{\begin{array}{l}
\dot{S}=-\rho S-\bar{A}(\dot{u})^{T} S-S \bar{A}(\dot{u})+\bar{C}^{T} \bar{C} \\
S(0)>0
\end{array}\right.
$$

with $\rho$ a positive constant and $R$ a diagonal matrix acting as a filter. In the noise-free case, $R=I_{n}$ (identity matrix of order $n$ ).

\section{B. Practical stability of the observer}

The time-varying perturbations $\gamma(t-h(t))$ and $\eta(t)$ prevent the asymptotic convergence of the observer error. Consequently, only a practical convergence to a ball of radius $r$ around the origin is achievable. The size of $r$ is tightly related to the size of the perturbation and the observer gain. The following lemma, given in [3], will be useful to formulate the main result.

Lemma 1: Consider that $S$ is defined by (9) and that Assumption 6 holds. Then there exists a real $\rho_{0}$ such that for any symmetric positive definite matrix $S(0)$, for all $\rho \geq \rho_{0}$, there exists $\bar{\alpha}>0, \bar{\beta}>0, t_{0}>0$ such that for all $t>t_{0}$

$$
\bar{\alpha} I_{n+1} \leq S(t) \leq \bar{\beta} I_{n+1}
$$

where $I_{n+1}$ is the identity matrix of order $n+1$.

Now, the main result can be stated.

Theorem 2: Consider system (6) and any input $u(t)$ and delay $h(t)$ such that Assumptions 1-6 are fulfilled. Then, there exist positive scalars $t_{0}, k, r, \theta$ such that for all $t \geq t_{0}$ the following inequality holds:

$$
\| \begin{aligned}
& \hat{X}(t)-X(t)\|\leq k\| e\left(t_{0}\right) \| \exp \left(-\theta\left(t-t_{0}\right)\right)+r . \\
& \text { Proof: }
\end{aligned}
$$

Define the Lyapunov candidate function as

$$
V(e)=e^{T} S e
$$

with $S$ given by (9) and $e=\hat{X}-X$, the error dynamics of the observer. The objective is to show that (11) complies with the assumptions of Lemma 9.4 in [15].

From Lemma 1, there exists $\rho_{0}$ and $t_{0}$ such that

$$
\bar{\alpha}\|e\|^{2} \leq V(e) \leq \bar{\beta}\|e\|^{2}
$$

for all $\rho \geq \rho_{0}$ and $t \geq t_{0}$. Furthermore, the dynamics of the observer of the undisturbed system is

$$
\dot{e}=\left[\bar{A}(\dot{u})-S^{-1} \bar{C}^{T} \bar{C}\right] e .
$$

Then, by using (9) and (13), the time derivative of (11) is

$$
\dot{V}(e)=-\rho e^{T} S e-e^{T} \bar{C}^{T} \bar{C} e .
$$

Since $e^{T} \bar{C}^{T} \bar{C} e \geq 0$, one has the inequality

$$
\dot{V}(e) \leq-\rho \bar{\alpha}\|e\|^{2} .
$$

In addition, $V$ satisfies the relation

$$
\left\|\frac{\partial V}{\partial e}\right\| \leq 2 \bar{\beta}\|e\| .
$$

Equations (12), (14) and (15) holds globally so Lemma 9.4 from [15] ensures that

$$
\|e(t)\| \leq k\left\|e\left(t_{0}\right)\right\| \exp \left(-\theta\left(t-t_{0}\right)\right)+r
$$

with $k=\sqrt{\frac{\bar{\beta}}{\bar{\alpha}}}, \theta=\frac{\rho \bar{\alpha}}{2 \beta}$ and

$$
r=\frac{2 \bar{\beta}^{2}}{\rho \bar{\alpha}^{2}} \sup _{t>t_{0}}\|\Gamma(t)\| .
$$

In a particular case, it is possible to evaluate the value of $r$.

Corollary 1: For constant delays and ramp inputs, the observation error converges exponentially to zero and one has $r=0$.

Proof: Assumptions 3 and 4 gives

$$
\sup _{t>t_{0}}\|\Gamma(t)\| \leq c_{1} \bar{h}^{2} M+c_{2} H
$$

with $c_{1}$ and $c_{2}$ stricly positive scalars. Furthermore, if the delay is constant then its dynamics is equal to zero so $H=0$; if the input is a ramp, its second time-derivative is 0 so $M=0$. As a consequence, the upper bound of $\Gamma$ is 0 and the radius $r$ given in (16) cancels which ends the proof.

Note that if the delay is slowly-varying then the approximation will be more accurate because $H$ will be smaller. Similarly, the smaller $M$ and $\bar{h}$, the finer the approximation (5) and the smaller the convergence radius $r$. Theoretically, it is possible to add higher order terms in the approximation (5) to reduce the uncertain term $\gamma$; however, it would require to compute high order time-derivatives of $u$. Observer (8) does not guarantee the boundedness of $\hat{h}$ to $[0, \bar{h}]$ so a projection of $\hat{h}$ on $[0, \bar{h}]$ can be made if the bounds are known [5][6][10]. Simulation results are provided in the next section to illustrate the efficiency and the limits of this new method. 


\section{Simulations}

\section{A. Model presentation and observer design}

A second order system has been chosen to illustrate previous result. Its input-output representation reads as

$$
\ddot{y}+\beta_{1} \dot{y}+\beta_{0} y=u(t-h(t)),
$$

and its state space representation is

$$
\left\{\begin{array}{l}
\dot{x}(t)=\left[\begin{array}{cc}
0 & 1 \\
-\beta_{0} & -\beta_{1}
\end{array}\right] x(t)+\left[\begin{array}{l}
0 \\
1
\end{array}\right] u(t-h(t)) \\
y(t)=C x(t)=x_{1}(t) .
\end{array}\right.
$$

The system is observable and the relative degree of $y$ equals two; Assumptions 1 and 2 hold. The parameters chosen for all the simulations are $x(0)=[1.5,1]^{T}, \beta_{1}=2, \beta_{2}=3$ and $h(t) \in[0,1]$. The extended system is defined by matrices:

$$
\bar{A}(\dot{u})=\left[\begin{array}{ccc}
0 & 1 & 0 \\
-\beta_{0} & -\beta_{1} & 0 \\
0 & 0 & -\dot{u}
\end{array}\right], \quad \bar{B}=\left[\begin{array}{l}
0 \\
1 \\
0
\end{array}\right] \text { and } \bar{C}=\left[\begin{array}{lll}
1 & 0 & 0
\end{array}\right] .
$$

The parameter $\rho$ is chosen equal to $5, S(0)=I_{3}$ (the identity matrix of dimension 3). The value of $\rho$ is a compromise between fast time-response and noise amplification (in the real case). The initial conditions of the observer are $\hat{x}(0)=$ $[0,0]^{T}$ and $\hat{h}(0)=0.4$.

\section{B. Noise-free simulations}

Two kinds of delay are used in the sequel:

- $h_{1}(t)=\left\{\begin{array}{l}0.15 \text { for } 0 \leq t \leq 15 \\ 0.6 \text { for } 15<t \leq 30 \\ 0.3 \text { otherwise }\end{array}\right.$

- $h_{2}(t)=0.4+0.2 \sin (0.4 t)$

The delay $h_{1}$ is a piecewise function whose each sub function complies with Assumption 3. The delay $h_{2}$ complies with Assumption 3. Two cases of input signals are tested:

- a ramp : $u_{1}(t)=0.2 t$

- a sinusoidal input: $u_{2}(t)=\sin (0.1 t)$

For the input $u_{1}$, one has $\dot{u}_{1}(t)=0.2$ and $\ddot{u}_{1}(t)=0$ so Assumption 4 and 6 are true. The input $u_{2}$ is regularly persistent because the condition (7) holds almost everywhere so Assumption 6 is true. Figure 1 displays inputs $u_{1}, u_{2}$ and their derivatives.

On Figure 2, the simulation is carried out with the delay $h_{1}$ and the input $u_{1}$. It can be noted that the observation errors tend to zero asymptotically (exponentially) because

- the delay dynamics $\eta(t)$ is 0 since the delay is piecewise constant;

- the Taylor approximation (5) is exact $u_{1}(t-h(t))=$ $u_{1}(t)-h(t) \dot{u}_{1}(t)$.

Consequently, the pertubation term $\Gamma$ in (6) is equal to 0 and the convergence radius is reduced to 0 . This result illustrates Corollary 1.

Figure 3 shows the result for the time-varying delay $h_{2}$ and the ramp input $u_{1}$. The Taylor approximation is still exact so $\gamma$ in (6) is equal to 0 . However, the observation error $x-\hat{x}$ does not tend to zero exponentially. Indeed, the observation error, $h-\hat{h}$, is introduced in the state observation.
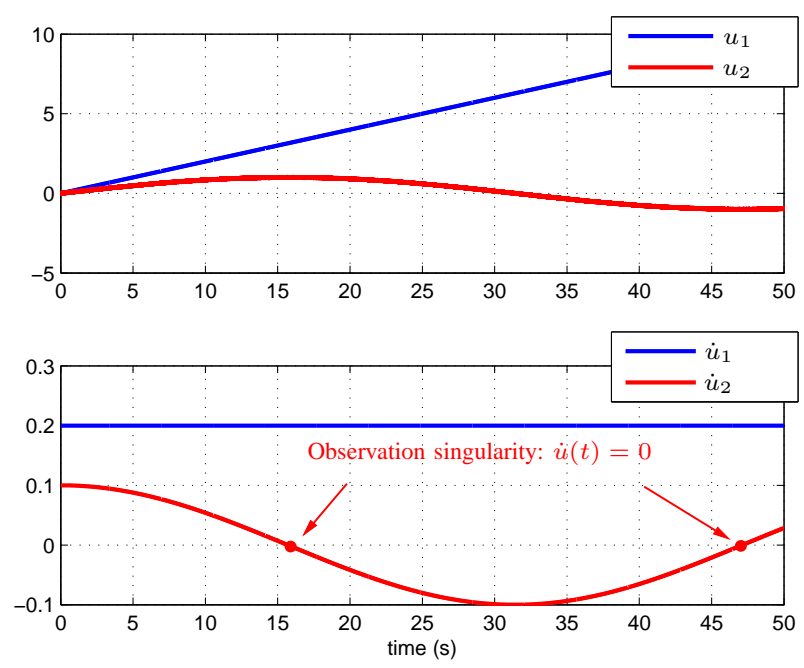

Fig. 1. Inputs and observation singularity
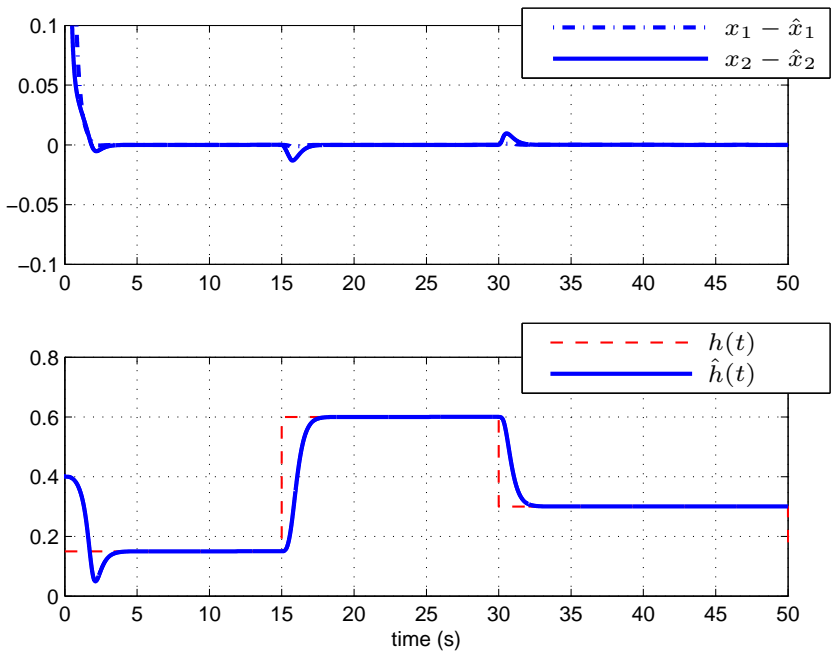

Fig. 2. Simulation 1 with $h(t)=h_{1}(t)$ and $u(t)=u_{1}(t)$ (ramp)

It is clear that $e_{x}=\hat{x}-x$ and $e_{h}=\hat{h}-h$ converge to a neighborhood around the origin and the size of this ball can be adjusted thanks to the gain $\rho$. Figure 4 shows this feature, with $\rho=15$, the convergence radius has decreased.

On Figure 5, the piecewise constant delay $h_{1}$ is associated to the sinusoidal input $u_{2}$. One can notice that the convergence radius for $e_{h}$ is smaller when $h$ is small. This is is mainly due to the accuracy of the approximation (5) that is better for small delays. The peaks are caused by the singularity in the observer gain $\left(S^{-1}\right)$ and the poor accuracy of the approximation (5) for large delays.

The last configuration with the sinusoidal input $u_{2}$ and the time varying-delay $h_{2}$ is presented on Figure 6. The analysis is the same as the one of simulations 2 and 3 :

- some peaks appear on the graph of $\hat{h}$ due to the observation singularity;

- the convergence radiuses for $e_{x}$ and $e_{h}$ are larger than 

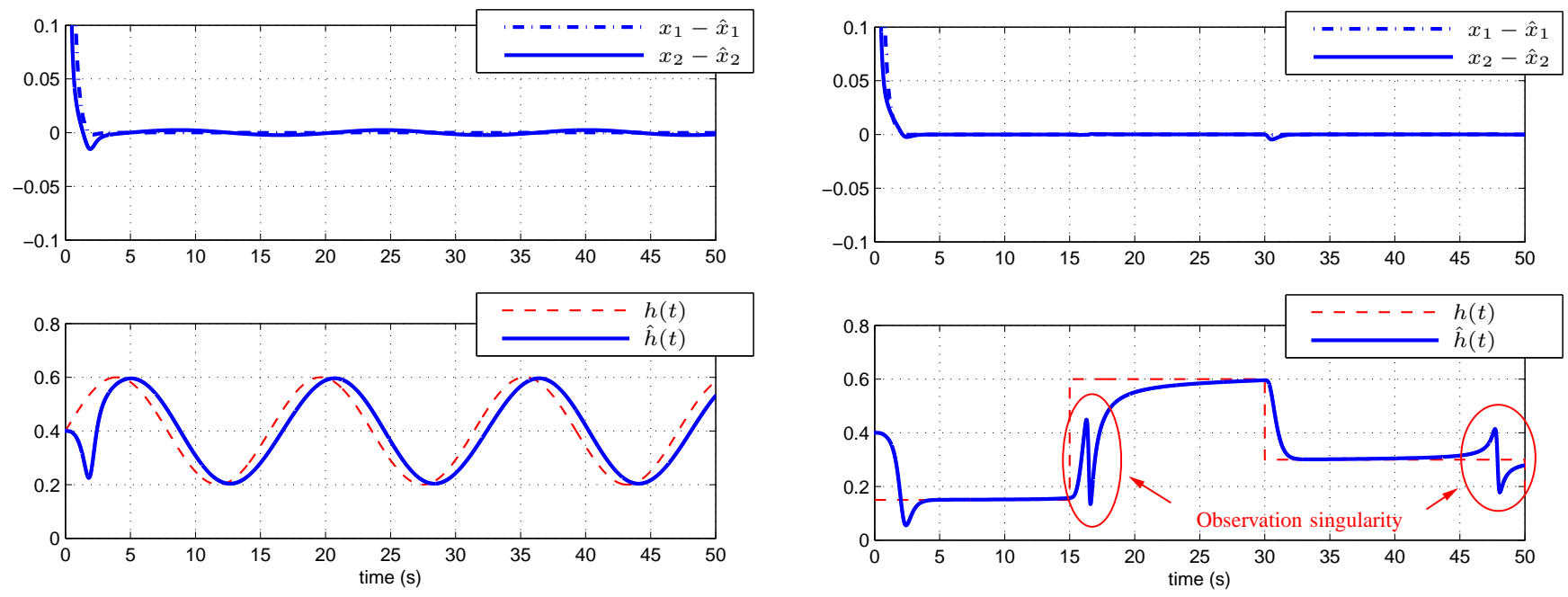

Fig. 3. Simulation 2 with $h(t)=h_{2}(t)$ and $u(t)=u_{1}(t)$ (ramp) and $\rho=5$
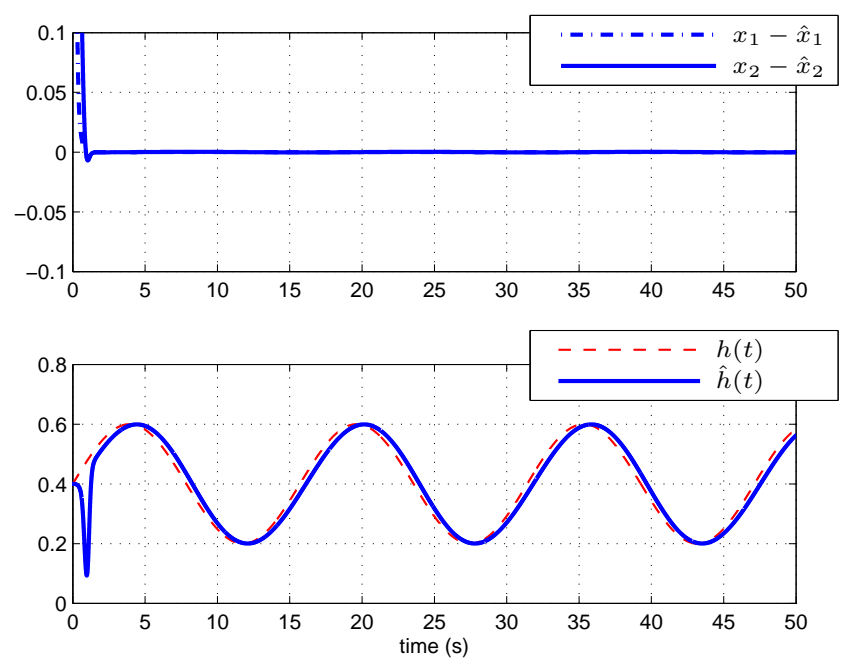

Fig. 5. Simulation 3 with $h(t)=h_{1}(t)$ and $u(t)=u_{2}(t)$ (sine)
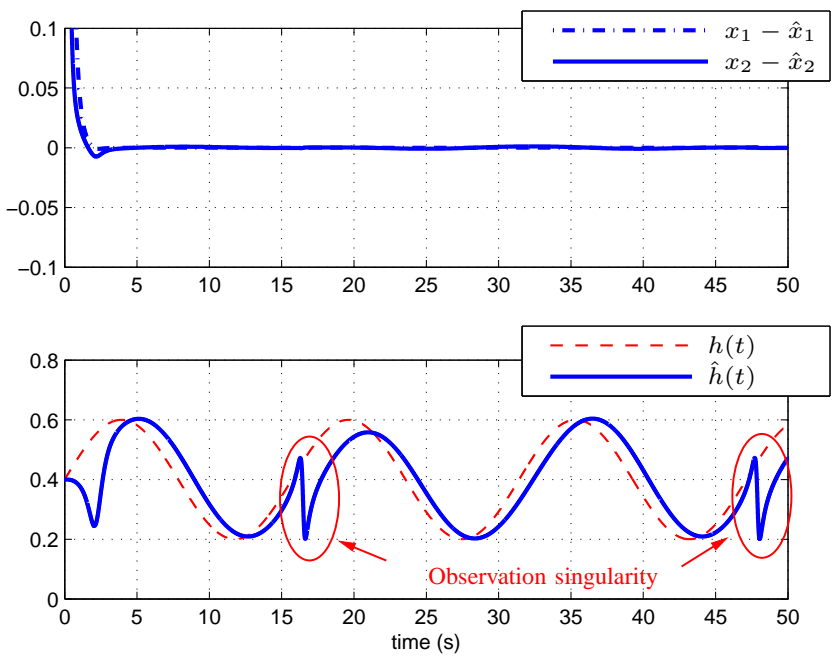

Fig. 6. Simulation 4 with $h(t)=h_{2}(t)$ and $u(t)=u_{2}(t)$ (sine)

Fig. 4. Simulation 2 with $h(t)=h_{2}(t)$ and $u(t)=u_{1}(t)$ (ramp) and $\rho=15$

for constant delay because of the term $\eta(t) \neq 0$.

Previous simulations have confirmed theoretical results. They illustrate the efficiency of the proposed robust observer technique

- to reconstruct the state of a system with an unknown and possibly time-varying delay in the input;

- to estimate the delay value.

The choice of the input is crucial. Indeed, the quality of the delay estimation highly depends on the input dynamics. As shown before, the ramp input is the best choice because it does not introduce any observation singularity and because the Taylor approximation is exact in this case. However, this is not always possible to apply it in practice. Consequently, a basic idea is to design inputs that are similar to a ramp and turned off the observer when it gets closer to the singularity

( $\dot{u}=0$ ). This method will be tested in the next subsection.

\section{Simulations with noisy measurement and noisy input}

In practice, the measurement and the input can be affected by noise. In the next simulation, a $5 \%$ white noise has been added to the output (measurement) and the input. A diagonal matrix $R$ has been implemented in the observer (see (8)) to filter the noise and a Levant differentiator [17] has been used to compute the input derivative. Furthermore, to overcome the observability singularity, the observer is turned off as soon as $|\dot{u}(t)| \leq \epsilon$. More precisely, only the delay-observer part of (8) is turned off, the state-observer part still runs. The parameter $\epsilon$ has to be tuned according to the input dynamics, in the next simulation $\epsilon=0.03$.

Figure 7 shows that the estimation accuracy is degraded but the observer still converges. Note also that the convergence is slower because of the filter. When the observer 
is turned off, the gain of the last equation in (8) is set to zero so $\dot{\hat{h}}=0$ that is why $\hat{h}$ is constant. Because of this observation, the technique is efficient when the delay varies slowly; the tuning of $\epsilon$ has to be a tradeoff between avoiding the singularity peaks and keeping an accurate estimation of $h$.
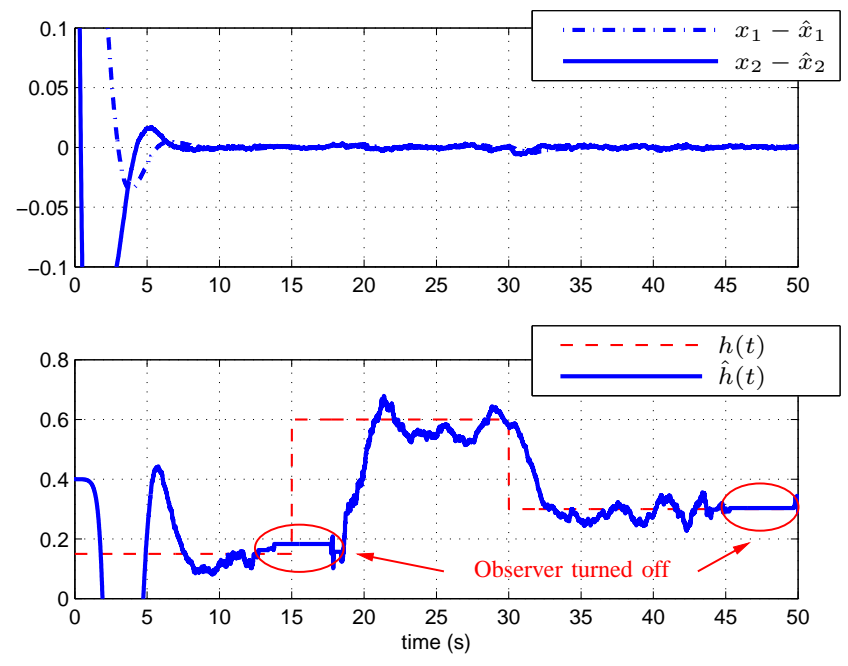

Fig. 7. Simulation 5 with $h(t)=h_{1}(t)$ and $u(t)=u_{2}(t)$ (sine) with measurement noise

\section{CONCLUSION}

This paper presents a new and original approach for observer design of input delay systems. This observation solution allows to estimate both state and delay (timevarying). The Taylor approximation is exploited to take out the delay from the retarded input. Then an extended system is created with the delay as a part of the augmented state. An observability condition is derived from the analysis of this extended system. Finally, a Kalman-like observer is design and practical stability is obtained. It is shown that asymptotic convergence can be achieved for constant delay with particular inputs. All the results are illustrated by numerous simulations.

The extension to nonlinear or MIMO systems as well as the observation in closed-loop are considered for future developments.

\section{REFERENCES}

[1] M. Agarwal and C. Canudas. On-line estimation of time delay and continuous-time process parameters. In American Control Conference, Seattle, USA, 1986.

[2] L. Belkoura, J-P. Richard, and M. Fliess. Parameters estimation of systems with delayed and structured entries. Automatica, 45(5):1117 $-1125,2009$.

[3] G. Besançon, G.Bornard, and H. Hammouri. Observer synthesis for a class of nonlinear control systems. European Journal of Control, 2(3):176 - 192, 1996.

[4] S. Björklund. A Survey and Comparison of Time-Delay Estimation Methods in Linear Systems. PhD thesis, Linköping University, Sweden, 2003.

[5] D. Bresch-Pietri and M. Krstic. Adaptive trajectory tracking despite unknown input delay and plant parameters. Automatica, 45(9):2074 2081, 2009.
[6] D. Bresch-Pietri and M. Krstic. Delay-adaptive predictor feedback for systems with unknown long actuator delay. IEEE Transactions on Automatic Control, 55(9):2106-2112, 2010.

[7] GC Clifford. Time delay estimation for passive sonar signal processing. IEEE Transactions on Acoustics, Speech and Signal Processing, 29(3):463-470, 1981.

[8] S. Diop, I. Kolmanovsky, P.E. Moraal, and M. van Nieuwstadt. Preserving stability-performance when facing an unknown time-delay. Control Engineering Practice, 9(12):1319 - 1325, 2001.

[9] S.V. Drakunov, W. Perruquetti, J.-P. Richard, and L. Belkoura. Delay identification in time-delay systems using variable structure observers. Annual Reviews in Control, 30(2):143 - 158, 2006.

[10] D.L. Gaudette and D.E. Miller. Stabilizing a SISO LTI plant with gain and delay margins as large as desired. IEEE Transactions on Automatic Control, 59(9):2324-2339, 2014.

[11] M. Ghanes, J. de Leon, and J. Barbot. Observer design for nonlinear systems under unknown time-varying delays. IEEE Transactions on Automatic Control, 58(6):1529-1534, 2013.

[12] H. Hammouri and J. de Leon Morales. Observer synthesis for stateaffine systems. In Conference on Decision and Control, Honolulu, USA, 1990.

[13] Robert Hermann and Arthur J Krener. Nonlinear controllability and observability. IEEE Transactions on automatic control, 22(5):728-740, 1977.

[14] J.P. Hespanha, P. Naghshtabrizi, and Yonggang Xu. A survey of recent results in networked control systems. Proceedings of the IEEE, 95(1):138-162, 2007.

[15] H.K. Khalil. Nonlinear Systems. Prentice Hall PTR, 2002.

[16] C. Knapp and G. Clifford Carter. The generalized correlation method for estimation of time delay. IEEE Transactions on Acoustics, Speech and Signal Processing, 24(4):320-327, 1976.

[17] A. Levant. Robust exact differentiation via sliding mode technique. Automatica, 34(3):379 - 384, 1998.

[18] A. Manitius and A.W. Olbrot. Finite spectrum assignment problem for systems with delays. IEEE Transactions on Automatic Control, 24(4):541-552, 1979.

[19] M. Nihtilä, T. Damak, and J.P. Babary. On-line estimation of the time delay via orthogonal collocation. Simulation Practice and Theory, 5(2):101 - 120, 1997.

[20] A O'Dwyer. A survey of techniques for the estimation and compensation of processes with time delay. Technical Report AOD.00.03, Dublin Institute of technology, 2000.

[21] A. Polyakov, D. Efimov, W. Perruquetti, and J.-P. Richard. Output stabilization of time-varying input delay systems using interval observation technique. Automatica, 49(11):3402 - 3410, 2013.

[22] A. Seuret, T. Floquet, J-P. Richard, and S. Spurgeon. Observer design for systems with non small and unknown time-varying delay. In Topics in Time Delay Systems, volume 388 of Lecture Notes in Control and Information Sciences, pages 233-242. Springer Berlin Heidelberg, 2009.

[23] O. J. M. Smith. Closer control of loops with dead time. Chemical Engineering Progress, 53(5):217-219, 1957.

[24] Y. Tipsuwan and M-Y. Chow. Control methodologies in networked control systems. Control Engineering Practice, 11(10):1099 - 1111, 2003.

[25] J. Tuch, A. Feuer, and Z.J. Palmor. Time delay estimation in continuous linear time-invariant systems. IEEE Transactions on Automatic Control, 39(4):823-827, 1994. 